\title{
Balancing functional and nutritional quality of oils and fats: Current requirements and future trends
}

\author{
Karen VAN DEN BREMT \\ Vera VAN HOED \\ Katrien MÜLLENDORFF \\ Filip ARNAUT \\ Puratos NV, Industrialaan 25, 1702 \\ Groot-Bijgaarden, Belgium \\ $<$ VandenBremtKaren@puratos.com $>$
}

\begin{abstract}
Oils and fats play an important role in the structure, aroma and stability of a wide variety of food products, as well as in their nutritional properties. For Puratos, a producer of ingredients for bakery, patisserie and chocolate sector, functionality and taste are of utmost importance, but the company also wants to contribute to the balanced diet of consumers. Vegetable oils and fats are used in margarines and releasing agents, vegetable creams, compound chocolate, fillings and emulsifiers. Each application requires an oil or fat with specific physicochemical properties in order to ensure the optimal structure, stability and taste of the end product. Traditionally, (partially) hydrogenated vegetable oils deliver important functional characteristics concerning crystallization behaviour, directly linked with the workability, melting properties, stability and mouth feel of the food product. However, due to negative nutritional implications, trans fats are to be replaced by healthier alternatives, preferably not by saturated fats. Consumers - and in some regions, legal instances - demand transfree or hydro-free products while not compromising on taste. Alternative fats and oils will be discussed concerning their functional and nutritional properties.
\end{abstract}

Key words: Oils \& fats, nutrition, health, functionality, hydrogenated fat, saturated fat
Consumer trends are there. The success of food products with nutritional benefits are the proof of it: low sugar soft drinks, high fibre containing breakfast cereals, omega 3 containing margarine spreads, etc. It is clear that consumers are nowadays paying more and more attention to their health and well-being. Consumers are starting to pay importance to what they eat. A growing number starts to read, understand and compare nutritional labels of different food products. This trend of increased consumer awareness on health and nutrition, has resulted in a growing demand for more nutritionally balanced food products. When improving this nutritional balance, salt, sugar and fat are by far the most important ingredients that are under attack. However, these ingredients often play an important functional role.

\section{Consumer preference: healthy food products but no compromise on taste}

Although the growing consumer awareness on health and nutrition, consumers do not want to make any compromise on taste when it comes to making choices for healthy alternatives. Especially viennoiserie and confectionary products bring a moment of real pleasure. They should in the first place be tasty. This is nowadays the challenge for many food companies: offering healthy, nutritionally balanced food products with a delicious taste. We are facing the challenge of eliminating or reducing ingredients that are less healthy and replacing them by more nutritious alternatives and this while keeping the functionality of the ingredients in the end product.
Puratos is an international group producing ingredients for the bakery, patisserie and chocolate sector with a specific focus on artisans, retailers and industrial clients. Puratos is world-wide a partner for many bakers, patissiers and chocolatiers in developing innovative products that respond to consumer trends. Through its vertically integrated production of enzymes, emulsifiers and sourdoughs, Puratos is able to offer unique knowledge on ingredients, as well as an expertise in applications and innovative solutions. Developing delicious tasting, nutritionally better balanced bakery, viennoiserie and confectionary products in line with the growing consumer trend is one of the main topics for Puratos. Nutrition and health is of the utmost importance to the company. Therefore Puratos commits to a nutrition charter stating

To cite this article : Van den Bremt K, Van Hoed V, Müllendorff K, Arnaut F. Balancing functional and nutritional quality of oils and fats: Current requirements and future trends. OCL $2012 ; 19(2): 83-88$. doi : 10.1684/ocl.2012.0441 
Table 1. Melting point of different fatty acids present in vegetable oils and fats. The structure of the fatty acid is strongly determining the melting temperature of the fatty acid. In general the melting point will increase with increasing chain length and will decrease with increasing number of unsaturations.

\begin{tabular}{|lll|}
\hline Abbreviated designation & Common name & Melting point $\left({ }^{\circ} \mathbf{C}\right)$ \\
\hline C4:0 & Butyric acid & -7.9 \\
C6:0 & Caproic acid & -3.9 \\
C8:0 & Caprylic acid & 16.3 \\
C10:0 & Capric acid & 31.3 \\
C12:0 & Lauric acid & 44.0 \\
C14:0 & Myristic acid & 54.4 \\
C16:0 & Palmitic acid & 62.9 \\
C18:0 & Stearic acid & 69.6 \\
C18:1 & Oleic acid & 13.4 \\
C18:2 & Linoleic acid & -5.0 \\
C18:3 & Linolenic acid & -11.0 \\
C20:0 & Arachidic acid & 75.4 \\
C22:0 & Behenic acid & 80.0 \\
\hline
\end{tabular}

that it wants to contribute to the balanced diet of consumers by optimizing the nutritional profile of Puratos products, by intensifying the scientific research collaborations in the area of nutrition and by sharing knowledge on nutrition with customers.

\section{Oils \& Fats: unique characteristics translated in unique functionalities}

Fat, whether it is margarine, shortening, butter or liquid oil, is a key ingredient in bakery, patisserie and cocoa products. Each fat is characterized by unique features, such as its origin, chemical composition, crystallization and melting behavior. These unique characteristics will have a strong influence on the functionality of the fat in the food product.

\section{Origin - chemical composition}

Oils \& fats originate from many different sources. They can be found in fruits, beans, seeds and nuts such as palm, coconut, soybean, cocoa, sunflower, peanut and many more. Each of these oils \& fats are characterized by a specific and very different chemical composition, mainly at the level of fatty acid composition. Lauric fats such as coconut oil and palm kernel oil are rich in lauric acid (C12:0) whereas palm oil is rich in palmitic acid (C16:0) and other fats are rich in $\mathrm{C} 18$ fatty acids. The length of the fatty acids will strongly determine the melting point of the fat (Scrimgeour, 2010) (table 1). Also the amount of unsaturated fatty acids will have an influence on the melting point. Liquid oils, like rapeseed oil, sunflower oil, olive oil, are rich in oleic (C18:1), linoleic (C18:2) and/or linolenic acid (C18:3).

The more unsaturated the fatty acids are, the lower their melting point will be as shown in table 1.

The botanical origin of oils and fats will determine the amount of saturated and unsaturated fatty acids in food products and will thus have a great impact on the nutritional value of these products.

Not only the presence of unsaturated fatty acids will influence the functionality of the oils \& fats, also the configuration of the double bond in the unsaturated fatty

Table 2. Recommended dietary intake for total fat and fatty acids, expressed in percentage of energy (\%E) in adults (WHO, 2008).

\begin{tabular}{|ll|}
\hline Fat/Fatty Acid & Amount \\
\hline Total fat & $20-35 \% \mathrm{E}$ \\
Saturated fatty acid & $10 \% \mathrm{E}$ \\
Mono-unsaturated fatty acid & By difference \\
Total poly-unsaturated fatty acid & $6-11 \% \mathrm{E}$ \\
Trans fatty acid & $<1 \% \mathrm{E}$ \\
\hline
\end{tabular}

acid will have an effect on its functional behavior. Unsaturated fatty acids can be in the cis or the trans configuration. A high amount of trans fatty acids will result in a fat characterized by a sharp melting profile in function of temperature and a high crystallization speed. However, these trans fatty acids have a negative health impact by lowering the "good" $\mathrm{HDL}$ cholesterol and increasing the "bad" LDL cholesterol and lipoprotein (a) levels in the blood (Katan et al., 1994). Even the way in which fatty acids are connected on to the glycerol backbone in the fat molecule will have an impact on its functional behavior. Fats containing high amounts of triglycerides composed of 3 saturated fatty acids will crystallize faster than other fats (Calliauw et al., 2010). Fats with triglycerides built up in a symmetrical way with 2 saturated fats on position 1 and 3 , and 1 unsaturated fatty acid on the $2^{\text {nd }}$, middle position, will tend to crystallize slow. Additionally they will be able to crystallize in different crystal forms, some more stable than others. Cocoa butter is high in symmetrical triglycerides. Therefore chocolate will need to be crystallized following a very specific cooling trajectory in order to get the fat crystals in the most stable form (Longchampt and Hartel, 2004).

\section{Oils \& Fats processing - chemical composition}

Next to their origin, the chemical composition of oils and fats can also be altered by different processing steps. By hydrogenating oils \& fats, the amount of saturated fatty acids, and in some cases the amount of trans fatty acids will strongly increase. By fractionation, hard fraction and liquid fraction can be separated and it is even possible to concentrate fats with high amounts of symmetrical triglycerides. Finally inter-esterification can re-shuffle the fatty acids of the fat molecules on the glycerol backbone and this in a random or a position-specific way.

\section{Functionality in food products}

Fat will determine texture and mouthfeel of the end product ( $\mathrm{O}^{\prime}$ Brien et al., 2000) (figure 1). The presence of high amounts of fat (up to 30-40\%) will bring a pleasant softness and short bite to sweet rich breads like brioche. Depending on the melting behavior of the fat, 


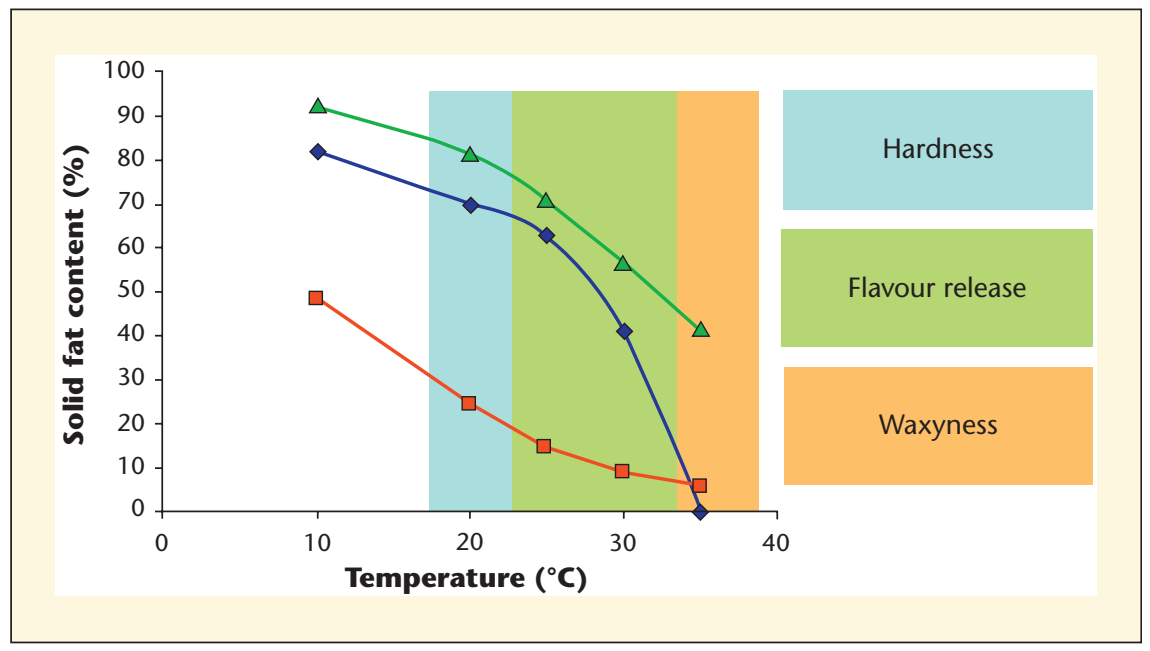

Figure 1. The melting behavior of oils and fats is expressed as Solid Fat Content (SFC)(in \%) in function of temperature $\left({ }^{\circ} \mathrm{C}\right)$. Different temperature zones can be distinguished in which the fat will have a different effect on the texture and mouthfeel of the food product. The ambient temperature zone will determine the hardness of the product. The temperature zone between 25 and $35^{\circ} \mathrm{C}$ is the zone in which the fat will melt and will have an effect on flavor release. The temperature zone around body temperature will determine if all fat will be melted in the mouth or whether still some fat will remain and will result in an unpleasant waxy fat film in the mouth when eating.

The blue curve is a typical curve of cocoa butter. Chocolate is hard at room temperature. When you bite in a bar of chocolate it will be very snappy. Once in your mouth the chocolate will quickly melt, giving an intense flavor release. At body temperature chocolate will be completely melted away.

The red curve is the melting curve of a fat that is soft at room temperature. It will slowly melt away leaving practically no solid fat at body temperature. This is a typical fat that can be used in soft chocolate fillings with a smooth, creamy melting texture sensation.

The green curve is the melting profile of a very hard fat that will slowly melt away and will still leave a large amount of solid fat at body temperature. When this fat would be used as such in a food product, it would give a very bad melting sensation and leave a waxy fat film in the mouth.

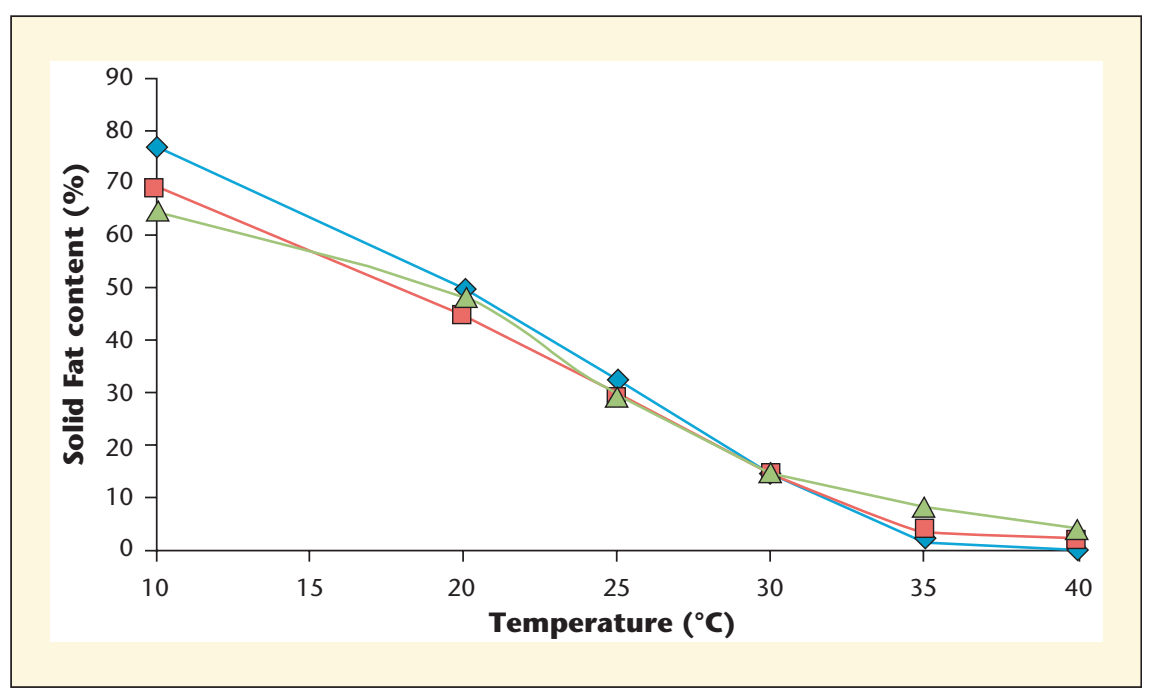

Figure 2. In order to improve the nutritional values of baked goods, Puratos has developed a complete range of margarines free from hydrogenated fats for all kind of applications: for incorporation in cake and rich breads, for preparation of fillings and also for laminated products such as croissants and puff pastry. This graph shows how alternative fats (green and red curve) were carefully selected to match the melting profile of the original hydrogenated, TFA containing fat (blue curve). However, not only the melting profile should perfectly match the original fat, also other functionalities like crystallization behavior are critical to obtain a nice and plastic lamination margarine. cocoa fillings can be creamy and smooth (Mohos, 2010). In this case fats are selected with a rather slow melting profile in function of the temperature. Fillings can also be very fast and cool melting. In this case lauric, often fractionated, fats are used or palm fractions with a high amount of symmetrical triglycerides.

Since fat is often a carrier for flavors, the presence and the type of fat will have a strong influence on the flavor release in foods products. However, the melting behavior of fats can also have a negative impact on the eating sensation of food products. Fats with a high melting point, often used for their heat resistance in warm climatological conditions, will leave an unpleasant, waxy fat film in the mouth, because the melting point of the fat is higher than the body temperature and thus the fat will not be able to melt away completely (figure 1).

Fat can determine the processing of the food products and consequently the eating sensation of the end product. Some fats will be very suitable for aerating and whipping fillings, giving them a fresh and light tasting sensation.

In the production of lamination margarine a careful selection of fats results in an optimal crystal structure of the margarine (Chrysan, 2010). This is essential for obtaining a plastic lamination margarine that can easily be laminated between the different layers of dough, resulting in a delicious crispy and flaky croissant, Danish or puff pastry.

Whipped products and margarines need numerous small ( $\beta^{\prime}$, beta prime) fat crystals to stabilize the emulsion (Chrysan, 2010). In contrast, the characteristic larger plate-like crystal form of cocoa butter give the chocolate the desired texture and strength, resulting in typical "snap" and mouth feel (Figoni, 2011).

\section{Oils \& Fats: a strong impact on health \& nutrition}

Last but not least fat will have an important impact on the nutritional values of the food products. Compared to other nutrients like proteins and carbohydrates, fats have a very high caloric value. According to the guidelines given by the World Health Organization (WHO, 2008) (table 2) only 20-35\% of our daily 


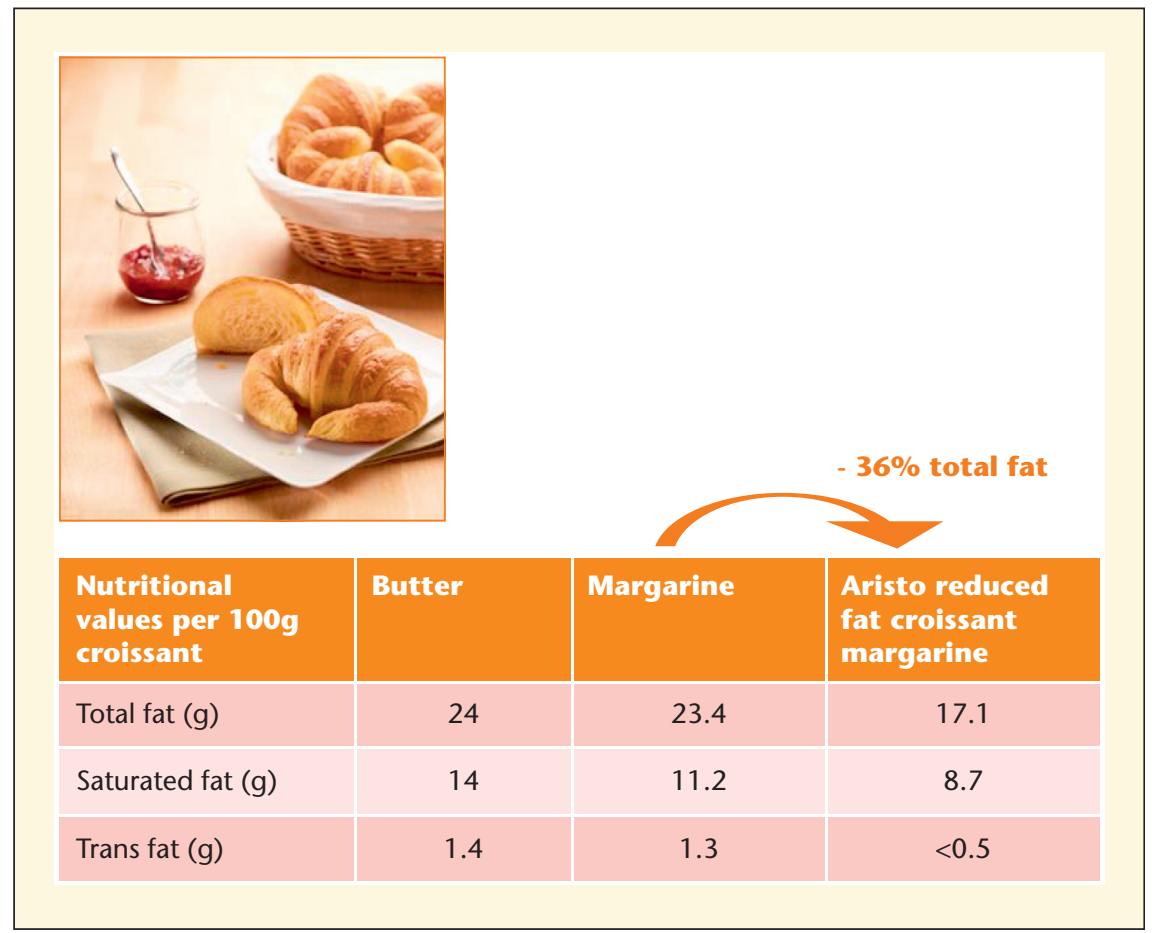

Figure 3. Aristo reduced fat croissants margarine enables bakers to make croissants that are up to $36 \%$ reduced in fat.

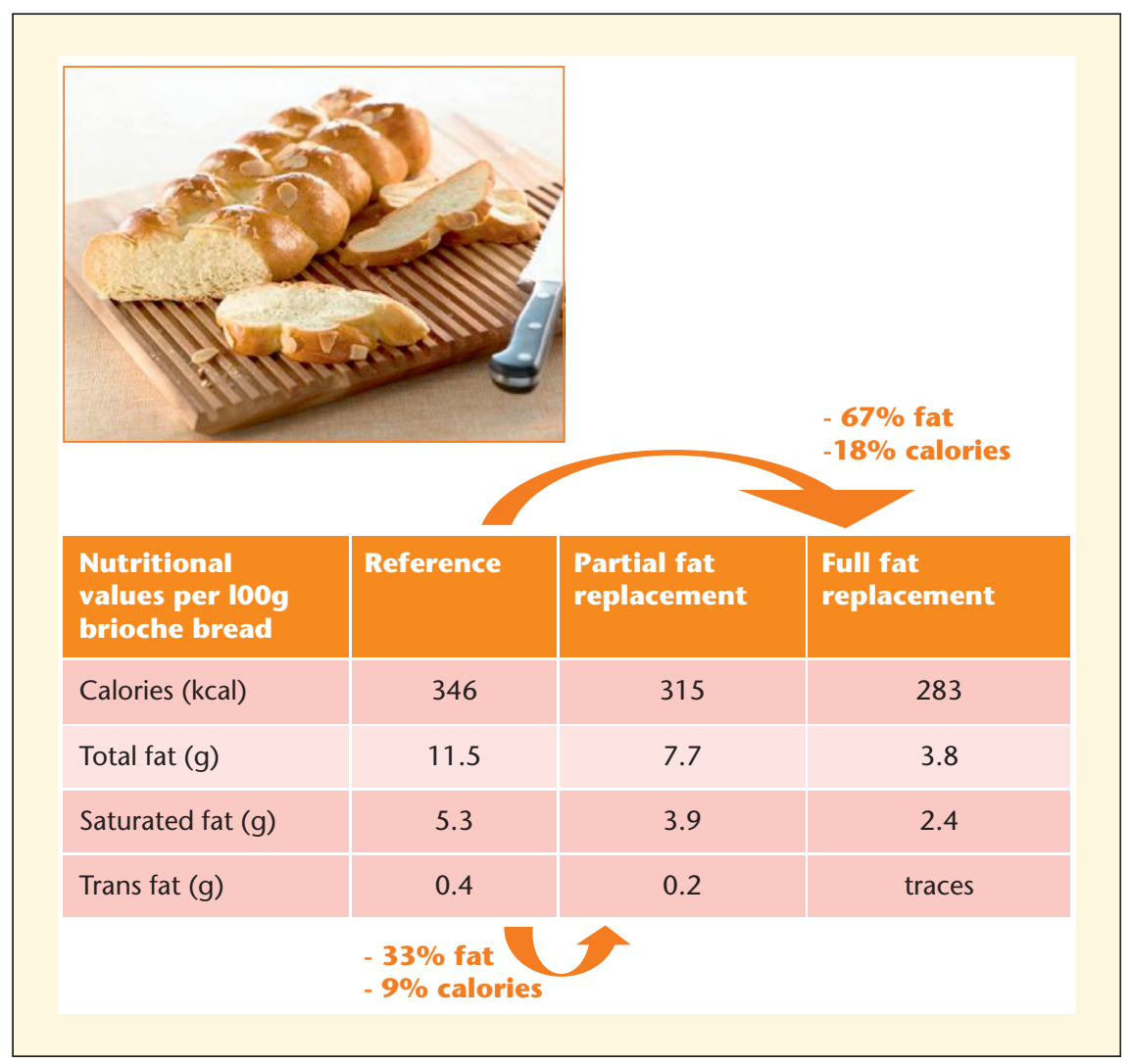

Figure 4. Puraslim reduces the amount of total and saturated fat in sweet, rich bread products such as brioche. In this example the nutritional values of a brioche bread prepared with $20 \%$ of margarine as a reference is compared with the nutritional values of a brioche bread in which half of the margarine or even all the margarine is replaced by Puraslim. A total fat reduction up to $67 \%$ can be obtained. recommended energy intake should come from fats. A guideline that is unfortunately in most mature countries exceeded with as a consequence a growing number of people with overweight or obesity as an eminent challenge for the future. Indeed, many of our food products contain high amounts of fat. Additionally, not only the total fat content but also the type of fats have an important health impact (Katan et al., 1994). Because of their ideal functionalities, like crystallization properties and structuring effect, a lot of fats used in food products contain high amounts of trans fatty acids and saturated fatty acids, both having a negative impact on cholesterol and related cardiovascular disease.

The consumer demand for nutritionally better balanced food products is the result of different driving forces that stimulate consumers for making more consciously the choice for a healthy lifestyle. In many countries governmental initiatives are taken to create consumer awareness for health and nutrition but also to discourage food companies to continue to use "bad" fats in their products. For example, since October 2011, a fat tax is in force in Denmark, which increases prices of food products that are rich in saturated fat (SAFA). For a product containing over $2.3 \%$ of SAFA, a tax of 2.15 euro per kg SAFA is raised. Next to the governmental initiatives, large international food companies, retail companies and quick service restaurants take themselves the initiative of improving the nutritional balance of their products.

\section{Balancing functionality, nutritional impact and consumer acceptance}

In this context producers of baked goods, patisserie and cocoa products are increasingly developing products that do not contain any hydrogenated fats or that are reduced in trans fatty acids, in saturated fatty acids or even reduced in total fat content. For each development the main challenge is to find the best solution for taking over the functionality of the original fats. This can be by using other fats but also by introducing new ingredients such as fibers, gums, proteins, starches, emulsifiers and more. 


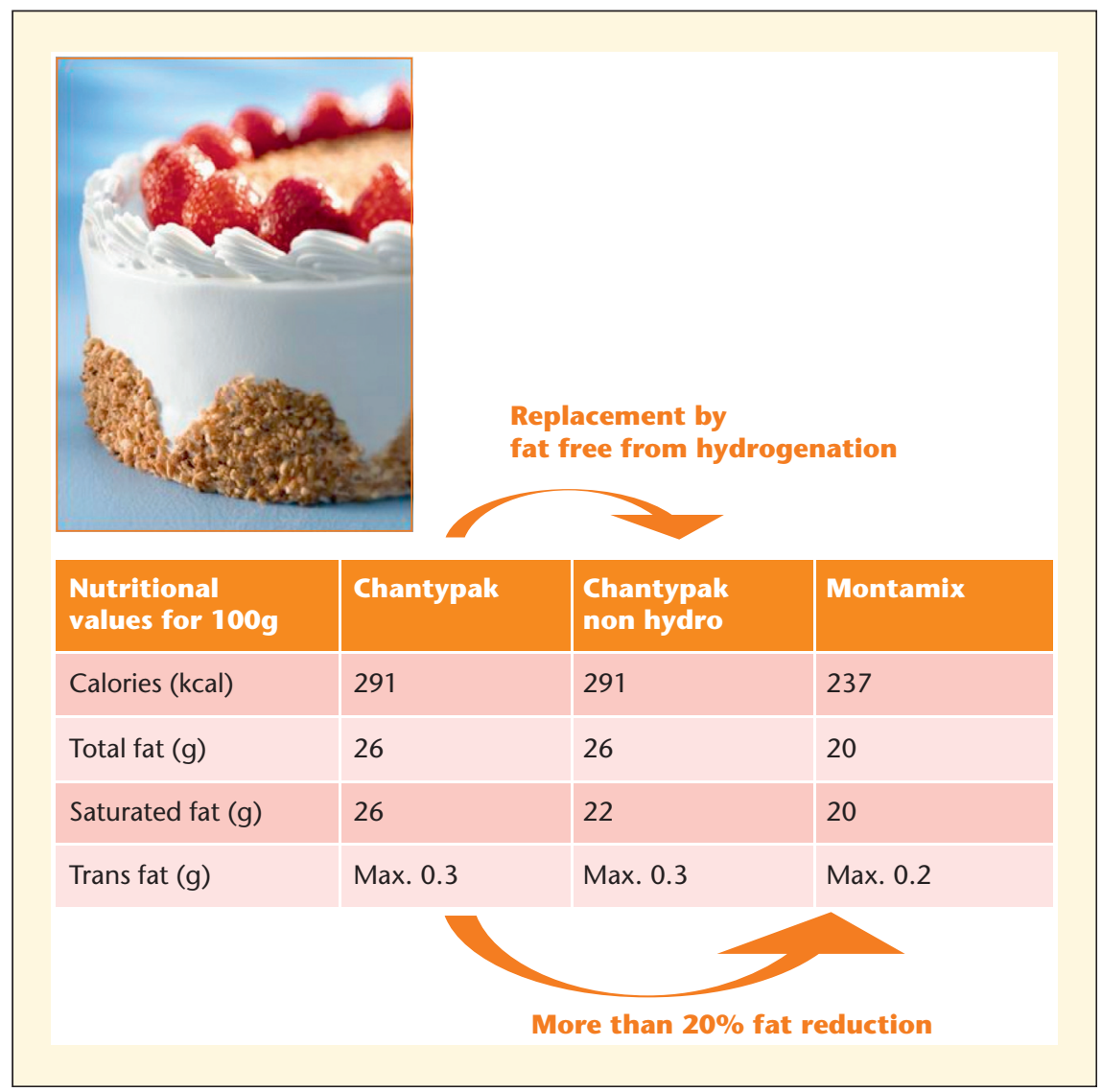

Figure 5. In each range of Puratos' non-dairy creams (Chantypak, Ambiante topping, Festipak...) products based on non-hydrogenated fats were developed. Also a non-dairy cream, Montamix, was developed that is reduced in fat.

For example, a complete range of margarines free from hydro fats was created by Puratos for all kind of applications, not only cream and cake but also for puff pastry and croissant. The replacement of hydro fats with nonhydro alternatives often has a strong impact on the properties and the taste of the finished product. Crystallization speed, hardness, melting properties and texture can be affected (Cavillot et al., 2009). In margarines for puff pastry, croissants and Danish, excellent plasticity and laminating properties were the challenging functionalities. The replacement towards non hydrogenated fats was achieved by carefully selecting other types of fats and emulsifiers, offering comparable crystallization properties and melting of the margarine (figure 2).

A margarine for laminated products such as croissants was developed to reduce the fat content of the croissants with $30 \%$. For this application the fat was replaced partially by inulin, offering soft and moist eating sensation of the sweet breads and cakes (figure 4).

Further, in the range of non-dairy toppings, different Puratos products were developed to reduce the amount of saturated fatty acids or to replace the hydrogenated fats by non hydrogenated fats. A non-dairy topping is an oil in water emulsion based on hydrogenated palm kernel oil. The fat content of a regular non-dairy cream is around $26 \%$ of fat.

Because the replacement of hydro fats with non-hydro alternatives often has a strong impact on the properties of the finished product (viscosity, whipping time, overrun and texture), different mixes of emulsifiers and stabilizers were used. In each range of Puratos' non-dairy creams (Chantypak, Ambiante topping, Festipak...) products based on nonhydrodrogenated fats were developed.

Next to the non-dairy creams free from hydrogenated fats, Montamix is a nondairy cream which was developed to reduce the fat with $20 \%$, meaning a $20 \%$ saturated fatty acid reduction. To compensate for the fat reduction, a different mix of stabilizers and emulsifiers was used (figure 5).

\section{Tasty nutritionally balanced products - a challenge for the food industry}

It is clear that each fat used in food products has been carefully chosen for its specific unique characteristics, resulting in a specific functionality in the food product. Replacing, reducing or even completely eliminating fats without losing that typical functionality in the food product is very challenging. Yet this typical functionality is the key for a delicious and tasty product.

Consumers are looking for more nutritionally balanced food products. It is a growing trend. However, they will not make any compromise on taste. For the food industry it will be the challenge for the future to respond to this growing consumer demand.

of the fat was replaced by a combined activity of emulsifiers, enzymes and flavor technology. Using this Puraslim, bakers are able to reduce the total fat content of their soft breads up to $70 \%$ and up to $40 \%$ for cake products. Consumer preference tests confirmed that this strong reduction of fat was achieved while keeping the pleasant,

\section{REFERENCES}

Calliauw G, Frederick E, Gibon V, De Greyt W, Wouters J, Foubert I, Dewettinck K. On the fractional crystallization of palm olein: solid solutions and eutectic solidification. Food Research International $2010 ; 43$ : 972-81. 
Cavillot V, Pierart C, Kervyn de Meerendré M, et al. Physicochemical properties of European bakery margarines with and without trans fatty acids. J Food Lipids 2009 ; 16 : 273-86.

Chrysan MM. Margarines and spreads. In: Shahidi F. Bailey's Industrial Oil and Fat Products, Sixth Edition. Volume 4, Chapter 2, 2010, Wiley-Interscience. Available online at: http://www.mrw.interscience.wiley.com/ biofp ISBN: 9780471678496; DOI: 10.1002/ 047167849X.

Figoni PI (ed.). How Baking Works: Exploring the Fundamentals of Baking Science. John Wiley \& Sons, 2011.
Katan MB, Zock PSL, Mensink RP. Effects of fats and fatty acids on blood lipids in hymans: an overview. Am / Clin Nutr 1994; 60 (Suppl.):1017S-22s.

Longchampt P, Hartel RW. Fat bloom in chocolate and compound coatings. Eur J Lipid Sci Technol 2004 ; 106 : 241-74.

O'Brien RD, Farr WE, Wan PJ, editors. Introduction to fats and oils technology. Second edition, Champaign, Illinois : AOCS press, 2000.

Mohos FA. Confectionery and chocolate engineering: principles and applications. John Wiley \& Sons, 2010.
Scrimgeour C. Chemistry of fats and oils. In: Shahidi F. Bailey's Industrial Oil and Fat Products, Sixth Edition. Volume 1, Chapter 1. Wiley-Interscience, 2010. Available online at http://www.mrw.interscience.wiley.com/ biofp ISBN: 9780471678496; DOI: 10.1002/ 047167849X.

WHO. Joint FAO/WHO Expert Consultation on Fats and Fatty Acids in Human Nutrition, Interim Summary of Conclusions and Dietary Recommendations on Total Fat \& Fatty Acids, 10-14 November, 2008, WHO, Geneva. (http://www.who.int/nutrition/topics/FFA_summary_rec_conclusion.pdf). 